Binghamton University

The Open Repository @ Binghamton (The ORB)

Mechanical Engineering Faculty Scholarship

Mechanical Engineering

$10-8-2018$

\title{
A Reliable MEMS Switch Using Electrostatic Levitation
}

Mark Pallay

Binghamton University--SUNY, mpallay1@binghamton.edu

Shahrzad Towfighian

Binghamton University--SUNY, stowfigh@binghamton.edu

Follow this and additional works at: https://orb.binghamton.edu/mechanical_fac

Part of the Mechanical Engineering Commons

\section{Recommended Citation}

M. Pallay \& S. Towfighian, A Reliable MEMS Switch Using Electrostatic Levitation, Applied Physics Letters, 113(22), pp. 213102, (2018); licensed under a Creative Commons Attribution (CC BY) license

This Article is brought to you for free and open access by the Mechanical Engineering at The Open Repository @ Binghamton (The ORB). It has been accepted for inclusion in Mechanical Engineering Faculty Scholarship by an authorized administrator of The Open Repository @ Binghamton (The ORB). For more information, please contact ORB@binghamton.edu. 


\title{
A Reliable MEMS Switch Using Electrostatic Levitation
}

\author{
Mark Pallay ${ }^{1}$ and Shahrzad Towfighian ${ }^{1, \text { a) }}$ \\ Mechanical Engineering Department, Binghamton University \\ 4400 Vestal Parkway East \\ Binghamton, New York, 13901
}

(Dated: October 8, 2018)

In this study an electrostatic MEMS beam is experimentally released from pull-in using electrostatic levitation. A MEMS cantilever with a parallel plate electrode configuration is pulled-in by applying a voltage above the pull-in threshold. Two more electrodes are fixed to the substrate on both sides of the beam to create electrostatic levitation. Large voltage pulses upwards of $100 \mathrm{~V}$ are applied to the side electrodes to release the pulled-in beam. A high voltage is needed to overcome the stronger parallel plate electrostatic force and stiction forces, which hold the beam in its pulled-in position. A relationship between bias voltage and release voltage is experimentally extracted. This method of releasing pulled in beams is shown to be reliable and repeatable without causing any major damage to the cantilever or electrodes. This is of great interest for any MEMS component that suffers from the pull-in instability, which is usually irreversible and permanently destroys the device, as it allows pulled-in structures to be released and reused. It also has a promising application in MEMS switches by opening up the possibility of a normally closed switch as opposed to current MEMS switches, which are normally open.

PACS numbers: Valid PACS appear here

Keywords: MEMS, Electrostatic Levitation, Pull-in instability, Release, Switch

\section{INTRODUCTION}

Microelectromechanical Systems (MEMS) are of key importance for a large number of commercial devices and are heavily relied upon to achieve the performance required by the manufacturer and consumer alike. These include smart-phones and computers, automobiles, airplanes, microscopes, laser printers, and much more, many of which would not be able to function properly without their MEMS components. With increasing demand for "smart" devices that can interact with the environment and their user, the demand for highly functional and reliable MEMS devices is expanding.

In application, most MEMS actuators and sensors utilize electrostatics to either induce or detect motion of a micro-structure. MEMS switches ${ }^{1-4}$, accelerometers $^{5,6}$, microphones $^{7,8}$, micro-mirrors ${ }^{9-13}$, and pressure sensors ${ }^{14}$ all use electrostatics to operate. The working principle behind electrostatic actuation can be explained through a parallel plate capacitor, where two parallel plates are given some initial charge to create an electric field between them. The electric field creates electrostatic forces that pull the two plates together. In MEMS, one of the plates is replaced with a small micro-structure, typically a beam or a plate, that can be pulled towards a fixed electrode by applying a voltage between them. This method of actuation allows precise control of its movement that is related to the profile of the electronic signal applied to the fixed electrode. Electrostatic forces are desirable because of their fast response time and simplicity in fabrication, however they

\footnotetext{
a) Electronic mail: stowfigh@binghamton.edu
}

also have drawbacks ${ }^{15}$.

One commonly undesirable phenomenon associated with electrostatic actuation is the pull-in instability. Pull-in occurs when the electrostatic force pulling the two electrodes together overcomes the mechanical forces separating them and the structure collapses. In many cases pull-in results in permanent damage to the device as the electrodes become stuck together and can not be separated even if the voltage is removed. The stiction forces such as van der Waals become much more significant at the micro-scale, and the parallel plate electrostatic force is only capable of pulling objects together, so release is often impossible ${ }^{15}$. Stiction can be mitigated by placing dimples on the bottom face of the beam or plate, thus reducing contact area and minimizing the stiction forces. However, even beams with dimples can frequently become stuck after pull-in, and therefore many electrostatic devices are designed to avoid pull-in entirely.

Much effort has been placed in creating electrostatic MEMS designs that do not experience pull-in at all. One of these methods is by actuating a structure using electrostatic levitation ${ }^{9-13,16-23}$. This involves a slightly different electrode configuration than the standard parallel plate design, with two extra electrodes that help induce an effectively repulsive force instead of an attractive one. This electrode configuration, first proposed by $\mathrm{He}$ and Ben $\operatorname{Mrad}^{9}$ for large travel ranges, is shown in Figure 1 for the case of a MEMS beam. The beam and fixed center electrode are kept at the same voltage level (typically ground), while the fixed electrodes on the side are supplied with a large voltage. When the beam is close enough to the center electrode, the electrostatic fringefield produced by the side electrodes pulls on the top of the beam more than the bottom, resulting in a net force upwards. It is not the case of a purely repulsive force that 


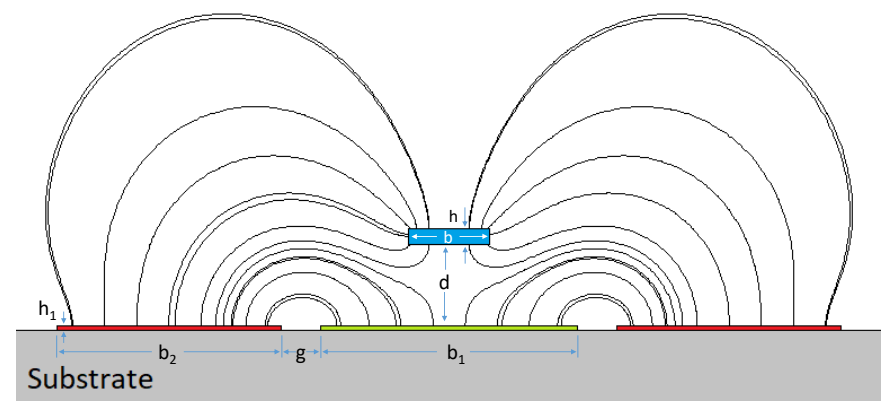

Figure 1. Repulsive force electrode configuration with electric field lines. The beam (top) and middle electrode are grounded, and side electrodes are charged (side voltage). The middle electrode can be given a DC voltage (bias voltage) to produce both attractive and repulsive forces on the beam simultaneously.

would occur between two positively charged particles, but rather an attractive force that acts in the opposite direction of the electrodes and is commonly referred to as repulsive to differentiate it from the attractive parallel plate force. The center electrode acts as a shield protecting the bottom face of the beam from the electric field and associated electrostatic force. As shown in Figure 1, some of the electric field lines that would have normally pulled on the bottom face of the beam are now traveling to the center electrode instead. The electric field at the top of the beam is relatively unaffected by the presence of the center electrode and therefore the direction of the net force becomes upward instead of downward when the beam-electrode gap is small.

If the beam is held to just one degree of freedom, which is common for thin and wide beams, it will not pull-in at all because the side electrodes are not in the beams path of motion. The center electrode will not create any attractive electrostatic forces on the beam because they are both at the same voltage potential, and thus pull-in will not occur. The authors have previously demonstrated in experiment that when excited with a harmonic voltage signal, the beam can collide with the center electrode, but instead of sticking it simply bounces off ${ }^{23}$.

A major drawback to electrostatic levitation is that it requires a very high voltage potential because it utilizes the weak fringe fields. To generate an electrostatic levitation force comparable to one generated by a standard parallel plate configuration, the voltage must be over an order of magnitude larger than the parallel plate voltage. In a previous study by the authors, voltages upwards of $150 \mathrm{~V}$ were applied to achieve around $10 \mu \mathrm{m}$ of static tip deflection for a $500 \mu \mathrm{m}$ long beam ${ }^{22}$. However, the large voltage potential and elimination of the pull-in instability allows repulsive actuators to move upwards of an order of magnitude greater than their initial gap ${ }^{22}$, as opposed to parallel plate actuators, which are typically limited by one third of the initial gap because of pull-in ${ }^{15}$.

Another advantage of electrostatic levitation is that it can be easily combined with parallel plate electrodes to enable bi-directional actuation ${ }^{24}$. Applying a bias to the middle electrode, along with the voltage on the side electrodes, creates both attractive and repulsive forces on the beam. The beam and middle electrode act as a parallel plate, while the side electrodes produce the levitation force. As with other bi-directional devices, such as a double parallel plate, bi-directional actuation requires multiple voltage inputs with each controlling the magnitude of the force in a single direction.

In this study, a MEMS beam is toggled between its pulled-in and released positions using a combination of parallel plate actuation and electrostatic levitation. A bias voltage is applied to the middle electrode to induce pull-in, then a high voltage pulse is applied to the side electrodes to release the beam from its pulled-in state. The authors demonstrate experimentally that the repulsive force is capable of overcoming the stiction forces holding the beam to the substrate. The capability of recovering from permanent pull-in failure of a MEMS structure is a great advancement and addresses a fundamental issue that has existed since the inception of electrostatically actuated MEMS. Thus it can help with making MEMS devices more reliable and reusable. It also opens the possibility of new applications for electrostatic MEMS by allowing them to utilize the pulled-in state as a functional element of the device, rather than a limitation. Almost all electrostatic MEMS are designed around pull-in and by using a combination of attractive and repulsive forcing this limitation can be relaxed or removed entirely. This has great potential for MEMS switches, as it can act as a normally closed switch $^{24}$, as opposed to current MEMS switches, which are normally open. It also has a promising application in micromechanical memories to read and erase bits as it can switch back and forth between two functional states; pulled-in and released ${ }^{25}$.

\section{A. Experimental Methods}

MEMS cantilevers are fabricated using PolyMUMPs standard fabrication by MEMSCAP ${ }^{26}$. An optical image of a fabricated beam is shown in Figure 2. The dimensions and material properties can be found in Table I. Dimples are placed on the bottom of the beam to reduce contact area and the associated stiction forces. While dimples can aid with release, the beams still suffer from stiction when pulled-in, as discussed in the next section. The cantilevers have the electrode layout shown in Figure 1. Images of both a pulled-in and released beam are shown in Figure 3. The beam can be modeled as an Euler-Bernoulli beam with electrostatic forcing, which can be calculated numerically with a 2D COMSOL simulation. A comparison of pure repulsive, pure attractive, and combined repulsive and attractive forces can be seen in Figure 4.

A schematic for the experimental setup is shown in 


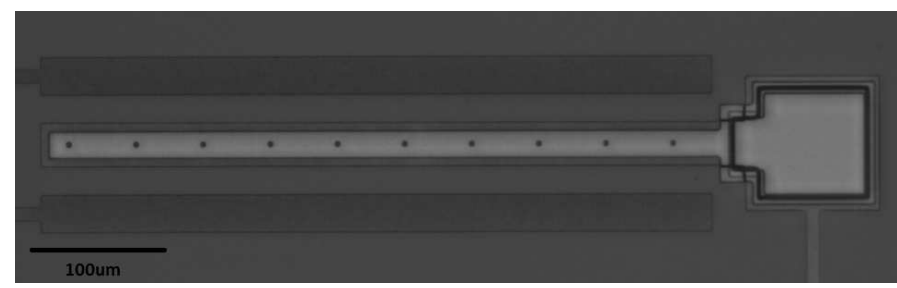

Figure 2. Optical image of a fabricated beam

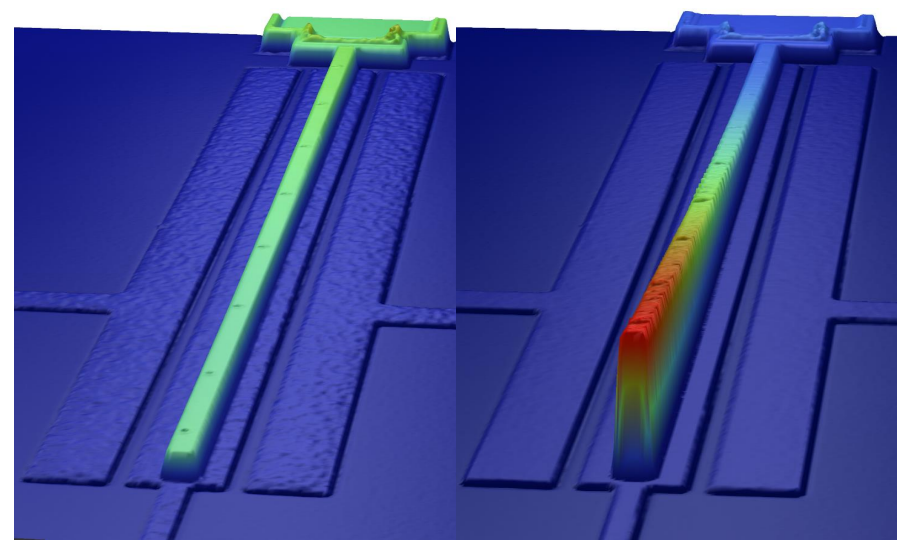

Figure 3. Image of the beam showing pull-in (left) at $2 \mathrm{~V}_{\text {bias }}$ and $0 \mathrm{~V}_{\text {side }}$, and release (right) at $2 \mathrm{~V}_{\text {bias }}$ and $120 \mathrm{~V}_{\text {side. }}$. The images were captured with a Wyko NT1100 Optical Profiler.

Figure 5. The cantilevers are placed in air and the tip displacement is measured with a Polytech MSA-500 Laser Vibrometer interfaced with MATLAB through a National Instruments USB 6366 Data Acquisition (DAQ). A B\&K Precision 9110 power supply and Krohn-Hite 7600 Wideband Power Amplifier supply the bias and side electrode voltage respectively. The bias voltage is measured directly with the DAQ, however the side voltage is well over the $10 \mathrm{~V}$ limitation of the DAQ and is measured with a Keithley 6514 electrometer, which is also con-

\begin{tabular}{lll}
\hline \hline Parameter & Variable & Value \\
\hline Beam Length & $\mathrm{L}$ & $500 \mu \mathrm{m}$ \\
Beam Width & $\mathrm{b}$ & $10 \mu \mathrm{m}$ \\
Beam Thickness & $\mathrm{h}$ & $2 \mu \mathrm{m}$ \\
Beam Anchor Height & $\mathrm{d}$ & $2 \mu \mathrm{m}$ \\
Side Electrode Gap & $\mathrm{g}$ & $5 \mu \mathrm{m}$ \\
Middle Electrode Width & $\mathrm{b}_{1}$ & $32 \mu \mathrm{m}$ \\
Side Electrode Width & $\mathrm{b}_{2}$ & $28 \mu \mathrm{m}$ \\
Electrode Thickness & $\mathrm{h}_{1}$ & $0.5 \mu \mathrm{m}$ \\
Dimple Length & $\mathrm{L}_{\mathrm{d}}$ & $0.75 \mu \mathrm{m}$ \\
Elastic Modulus & $\mathrm{E}$ & $150 \mathrm{GPa}$ \\
Density & $\rho$ & $2330 \mathrm{~kg} / \mathrm{m}^{3}$ \\
Poisson's Ratio & $\mathrm{v}$ & 0.22 \\
\hline \hline
\end{tabular}

Table I. Beam parameters

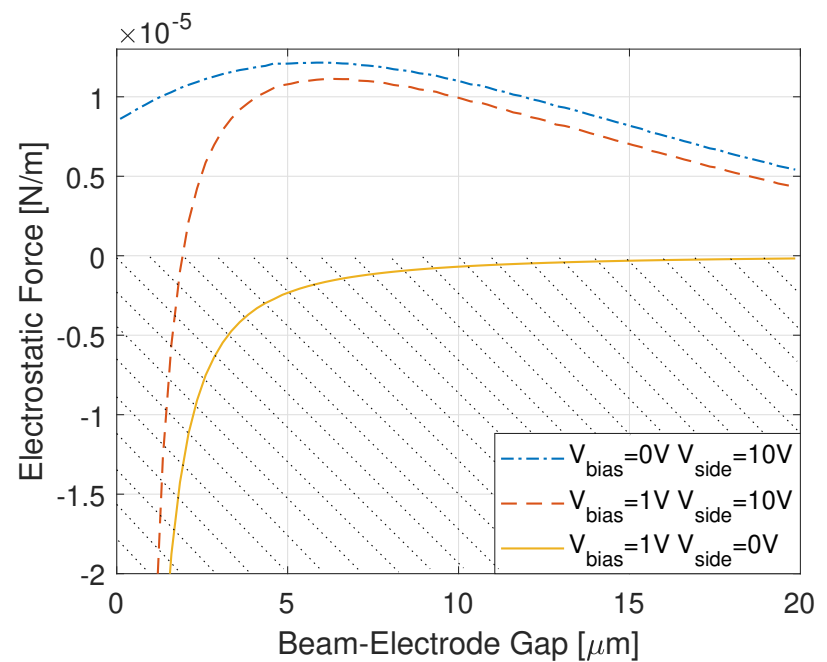

Figure 4. Electrostatic force on the beam versus gap distance simulated in COMSOL. The dashed area shows the attractive regime and the rest is the repulsive regime. The combined force with bias and side voltages behaves similar to the attractive force at low gaps and the repulsive force at large gaps. Applying $10 \mathrm{~V}$ on the side electrodes can change the force from attractive to repulsive outside of very small gaps.

\section{trolled with MATLAB.}

In the experiment a bias voltage is applied to the center electrode to start the beam in its pulled-in position. The bias is then adjusted to a specified level and held constant before a series of short, high voltage pulses are applied to the side electrodes. The beam displacement is observed to determine whether or not the beam was released during the voltage pulses. A relationship between bias voltage and release side voltage is obtained to demonstrate the working principle of the repulsive switch.

\section{B. Results and Discussion}

Figure 6 shows the recorded switch motion and applied voltages. The bias voltage is initially set at $0 \mathrm{~V}$, then is increased to $4.5 \mathrm{~V}$, the pull-in voltage, and held constant. As the bias voltage ramps up the beam begins to respond before suddenly pulling in, which can be observed at approximately $1.2 \mathrm{~s}$. Two pulses of $195 \mathrm{~V}$ are applied to the sides after the beam is in the pulled-in position. The cantilever releases during both pulses, which can be observed jumping up to $20 \mu \mathrm{m}$ in the displacement signal. When the side voltage drops back to zero the beam immediately pulls back in and sticks to the substrate. The beams can be toggled to and from pull-in many times without failure. Even after failure no obvious mechanical damage is observed on the beam. The beam can be repeatedly and reliably toggled to and from the pulled-in position, without damaging the device, by applying and removing a voltage on the side electrodes. The bias volt- 


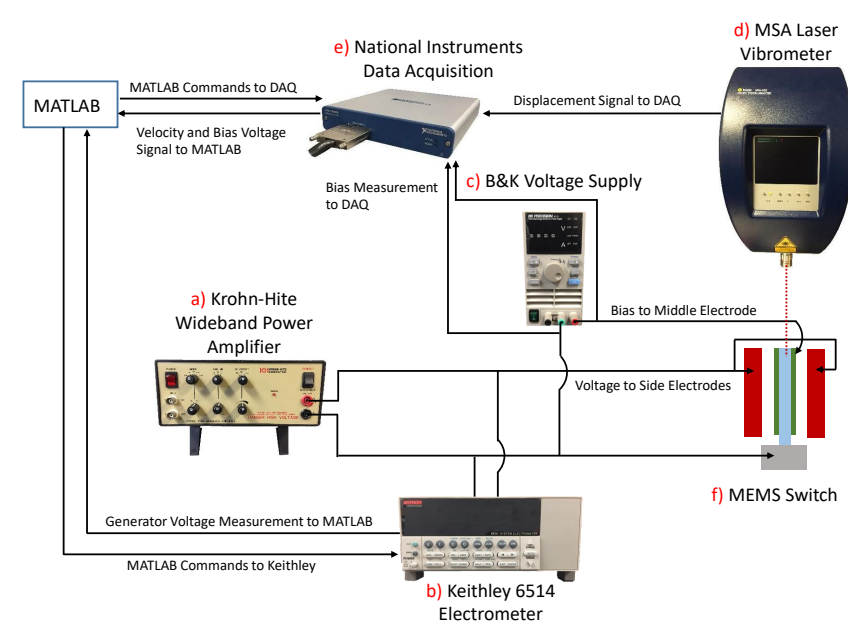

Figure 5. Experimental setup with a) Krohn-Hite 7600 Wideband Power Amplifier, b) Keithley 6514 Electrometer, c) B\&K Precision 9110 Power Supply, d) Polytech MSA-500 Laser Vibrometer, e) NI USB 6366 Data Acquisition, and f) the MEMS repulsive switch. The DAQ and electrometer are interfaced with MATLAB.

age determines the minimum side voltage needed to open the switch.

The experiment was repeated by adjusting the bias voltage and determining the associated release voltage. Figure 7 shows the release voltage for various bias levels. For biases that are less than the $4.5 \mathrm{~V}$ pull-in voltage, first pull-in is initiated at $4.5 \mathrm{~V}$, then the bias is reduced to the specified level. When the bias voltage is removed completely, the beam continues to stick to the middle electrode and $70 \mathrm{~V}$ is required to release the beam. At the pull-in voltage, $195 \mathrm{~V}$ is needed for release. Because of limitations with the PolyMUMPs chips voltages above $200 \mathrm{~V}$ were not applied.

Figure 7 demonstrates that the release voltage can be adjusted by changing the bias voltage. This is useful for a MEMS switch, which can be tuned to open at different threshold voltage levels. If paired with a transducer that is converting mechanical energy to electrical energy, the entire system can be designed to trigger the opening of a switch when the input passes a threshold ${ }^{24}$. In addition to the tunability, it also can act as a normallyclosed switch, which is not possible with a standard twoelectrode parallel plate configuration.

\section{Conclusion}

In this paper a MEMS cantilever is experimentally released from its pulled-in position using electrostatic levitation. This method provides a safe and effective way of releasing and reusing pulled-in MEMS beams, which would have otherwise been permanently stuck to the substrate, rendering them unusable. The obtained results are very promising for the field of MEMS research by increasing the longevity MEMS beams and allowing researchers to salvage and reuse devices that would have been discarded. It was also demonstrated that the release voltage can be controlled by changing the bias voltage, which opens up the possibility of a tunable, normallyclosed and bi-directional MEMS switch. Combining parallel plate actuation with electrostatic levitation allows for more robust MEMS devices while also increasing functionality for new MEMS sensors and switches that can overcome limitations of current designs.

\section{Acknowledgment}

This research was funded by NSF ECCS grant \#1608692..

\section{REFERENCES}

${ }^{1}$ A. Ramini, M. Younis, and Q. Su, Smart Materials and Structures 22, 025006 (13 pp.) (2013).

${ }^{2}$ H. M. Ouakad and M. I. Younis, Mathematical Problems in Engineering 2013 (2013).

${ }^{3}$ A. Bouchaala, N. Jaber, O. Shekhah, V. Chernikova, M. Eddaoudi, and M. Younis, Applied Physics Letters 109, 013502 (4 pp.) (2016).

${ }^{4}$ N. Jaber, S. Ilyas, O. Shekhah, M. Eddaoudi, and M. I. Younis, Journal of Microelectromechanical Systems (2018).

${ }^{5}$ M. I. Ibrahim and M. I. Younis, 2008 Proceedings of the ASME International Mechanical Engineering Congress and Exposition , 241 (2009).

${ }^{6}$ M. Sharma, E. H. Sarraf, and E. Cretu, Proceedings of the IEEE International Conference on Micro Electro Mechanical Systems (MEMS), 617 (2011).

${ }^{7}$ R. N. Miles, D. Robert, and R. R. Hoy, The Journal of the Acoustical Society of America 98, 3059 (1995).

${ }^{8}$ R. N. Miles, W. Cui, Q. T. Su, and D. Homentcovschi, Journal of Microelectromechanical Systems 24, 241 (2015).

${ }^{9} \mathrm{~S}$. He and R. Ben Mrad, IEEE Transactions on Industrial Electronics 52, 974 (2005).

${ }^{10} \mathrm{~S}$. He and R. Ben Mrad, Journal of Microelectromechanical Systems 17, 532 (2008).

${ }^{11} \mathrm{C}$. Fan and S. He, Journal of Microelectromechanical Systems 24, 2049 (2015).

${ }^{12}$ J. Chong, S. He, and R. Ben Mrad, IEEE/ASME International Conference on Advanced Intelligent Mechatronics, AIM , 1005 (2010).

${ }^{13}$ M. Ozdogan, M. Daeichin, A. Ramini, and S. Towfighian, Sensors and Actuators A: Physical, 265, 20 (2017).

${ }^{14}$ M. H. Hasan, F. M. Alsaleem, and H. M. Ouakad, Journal of Micromechanics and Microengineering 28 (2018).

${ }^{15}$ M. I. Younis, Mems Linear and Nonlinear Statics and Dynamics (Springer, New York, 2011).

${ }^{16}$ K. B. Lee and Y. H. Cho, Journal of Microelectromechanical Systems 10, 128 (2001).

${ }^{17}$ T. Sugimoto, K. Nonaka, and M. N. Horenstein, Journal of Microelectromechanical Systems 14, 718 (2005).

${ }^{18}$ N. Shen and E. Kan, Technical Digest. MEMS 2002 IEEE International Conference. Fifteenth IEEE International Conference on Micro Electro Mechanical Systems (Cat. No.02CH37266) , 598 (2002).

${ }^{19} \mathrm{~S}$. He and R. Ben Mrad, IECON Proceedings (Industrial Electronics Conference) , 4020 (2009).

${ }^{20}$ G. Li, X. Guo, Q. Zhao, and J. Hu, , 1095 (2015). 

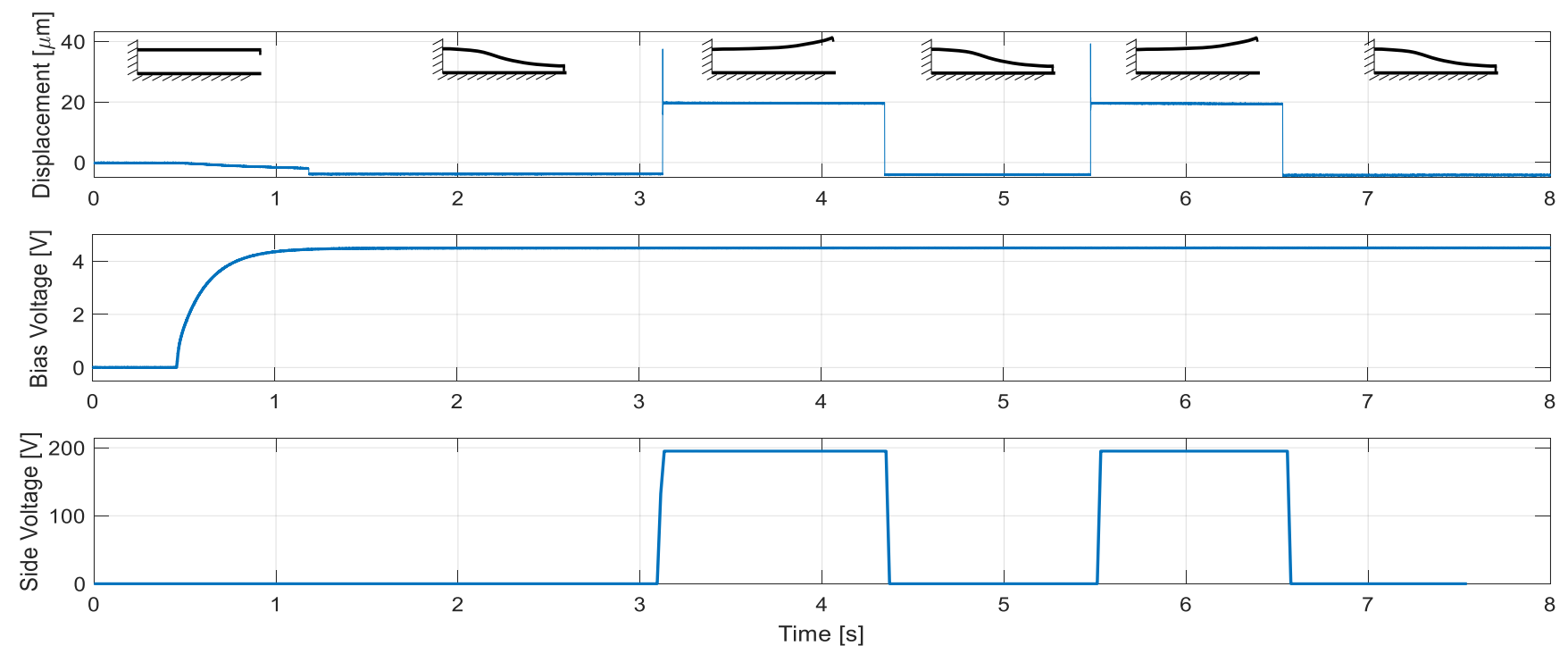

Figure 6. Measured beam tip displacement, bias voltage, and side voltage versus time. The beam pulls in as the bias voltage ramps up to $4.5 \mathrm{~V}$ and is released by supplying $195 \mathrm{~V}$ on the side. The illustrations at the top of the displacement signal roughly show the profile of the beam during each phase of the experiment.

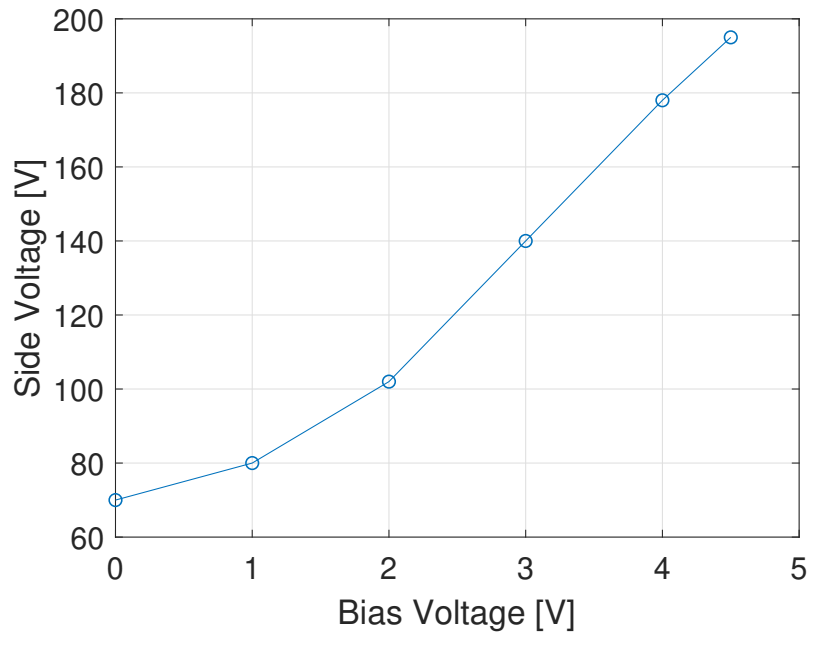

Figure 7. Bias voltage versus threshold release voltage.
${ }^{21}$ D. Y. Qiao, W. Z. Yuan, and X. Y. Li, Proceedings of 1st IEEE International Conference on Nano Micro Engineered and Molecular Systems, 1st IEEE-NEMS , 168 (2006).

${ }^{22}$ M. Pallay, M. Daeichin, and S. Towfighian, Nonlinear Dynamics 89, 1525 (2016).

${ }^{23}$ M. Pallay and S. Towfighian, Sensors and Actuators A: Physical 277, 134 (2018)

${ }^{24}$ M. Pallay and S. Towfighian, Proceedings of the ASME 2018 International Design Engineering Technical Conferences, Aug 26-29, 2018, Quebec City, Canada, DETC2018-85543, 8 pages (2018).

${ }^{25}$ D. Roodenburg, J. Spronck, H. Van der Zant, and W. Venstra, Applied Physics Letters 94, 183501 (2009).

${ }^{26}$ A. Cowen, B. Hardy, R. Mahadevan, and S. Wilcenski, "PolyMUMPs Design Handbook a MUMPs@ process," (2011). 